\title{
Comprehensive electronic structure characterization of pristine and nitrogen/phosphorus doped carbon nanocages
}

\author{
Hui Zhang ${ }^{1,2}$, Xin Li $^{2,3}$, Duo Zhang ${ }^{1,2}$, Liang Zhang ${ }^{2,4}$, Mukes Kapilashrami ${ }^{2,5}$, Sun Tao ${ }^{6}$, Per-Anders $^{2}$

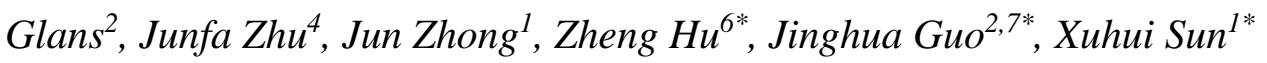

${ }^{1}$ Soochow University-Western University Centre for Synchrotron Radiation Research, Institute of Functional Nano and Soft Material (FUNSOM), Soochow University, Suzhou 215123, China

${ }^{2}$ Advanced Light Source, Lawrence Berkeley National Laboratory, Berkeley, CA 94720, USA

${ }^{3}$ Department of Theoretical Chemistry and Biology, School of Biotechnology, Royal Institute of Technology, S-10691 Stockholm, Sweden

${ }^{4}$ National Synchrotron Radiation Laboratory, University of Science and Technology of China, Hefei 230029, China

${ }^{5}$ Center For Engineering Concepts Development, Department of Mechanical Engineering, University of Maryland, College Park, MD 20742, USA

${ }^{6}$ Key Laboratory of Mesoscopic Chemistry of MOE, School of Chemistry and Chemical Engineering, Nanjing University, Nanjing 210093, China

\footnotetext{
${ }^{7}$ Department of Chemistry and Biochemistry, University of California, Santa Cruz, CA 95064, USA
}

\footnotetext{
*Corresponding author. Email: xhsun@ suda.edu.cn (Xuhui Sun), iguo@lbl.gov (Jinghua Guo), zhenghu@ nju.edu.cn (Zheng Hu) 1 
Abstract:

The electronic structures of carbon nanocages (CNCs) and nitrogen/phosphorus doped carbon nanocages (N-CNCs/P-CNCs) have been studied by X-ray photoelectron spectroscopy (XPS), X-ray absorption spectroscopy (XAS), X-ray emission spectroscopy (XES) and resonant X-ray emission spectroscopy (RXES). The doping configurations for N/P dopants are identified from the experiments. The results have shown that there are three major doping configurations for nitrogen but only one doping configuration for phosphorus. The nitrogen doping reveals the complex coexistence of graphite-like, pyridine-like and pyrrole-like configurations that are proved by density functional theory (DFT) simulations, while the phosphorus doping presents only the "graphite-like" configuration. The different configuration profiles result in less atomic structure ordering of N-CNCs than that of P-CNCs. XAS spectra obtained from both surface and bulk sensitive detection suggest different chemical environments between the interior and shell for all types of nanocages. The electronic structure modifications show significant difference between nitrogen and phosphorus doping from the DOS calculations. 


\section{Introduction}

Nanomatrices solely comprised of carbon atoms, e.g. carbon buckyballs $\left(\mathrm{C}_{60}\right)$, carbon nanotubes $(\mathrm{CNTs})$, and graphene, demonstrate remarkable physical and chemical properties by the virtue of large surface area and high charger-transfer rate, $[1,2]$ which are the two essential properties in the applications related to the energy storage and conversion.[3] For instance, graphene and CNTs have been widely used in Li-ion batteries due to their high surface area and superior electric conductivity.[4,5]

Non-metal doping into the carbon matrices introduces both lattice defects and disparity in the charge distribution between the constituents, which has been undertaken as a route to design the promising potential non-metal electrocatalyst for oxygen reduction reactions (ORRs)[6,7] and supporting materials for other catalysts.[8,9] Generally, the foreign dopants induce covalent bonds with the carbon matrices. The covalent functionalization accompanies a permanent alteration of the local chemical structure which substantially modifies both the structural and electronic physical properties. One such example is the boron doped graphene wherein three valence electrons of the dopant participate in $\sigma$ bonding with the surrounding carbon atoms, while the absence of $\pi$ electrons leads to a p-type doping effect. [10]

Different from $\mathrm{CNTs}_{\text {and }} \mathrm{C}_{60}$, carbon nanocages $(\mathrm{CNC})$ present a hollow cage-like morphology, with a few tens of nanometers in size for the cage and several nanometers for the shell thickness (Fig. 1A). The shells of nanocages are mostly presented as graphite-like layer structure. Although carbon nanocages (both pristine and doped) have previously been extensively evaluated for their potential implementation in applications related to alternative energy,[11-16] there is lack of a comprehensive understanding in regards to the electronic structure, local charge symmetries as well as the corresponding changes upon doping. Also, the different content of doping normally show various physical properties, such as $\mathrm{N}$ doped CNCs with different doping concentration for ORR catalyst[11] and electrode of supercapacitor[17]. Moreover, in light of the continuous advancements in the development of more complex carbon 
allotropes, there is a strong need to elucidate the electronic properties of these nanomatrices in order to further optimize their functional properties and performance in the applications that ultimately relay on the effective charge storage and transportation.

X-ray absorption spectroscopy (XAS) and X-ray emission spectroscopy (XES) offer the capability to project both the partial density of unoccupied and occupied states by detailing the local charge- and chemical- symmetry surrounding the constituents in matter. These characterization techniques have today established their prominence in the fields of nanomaterials science since they enable the evaluation of matter at the atomistic/molecular level with high precision and element selectivity, which arise from the excitation of a core-electron into the unoccupied electronic states and de-excitation of a valence-electron into the core vacancy.[18,19] In addition to the inherent elemental selectivity of X-ray, resonant X-ray emission spectroscopy (RXES) allows separation of the features that pertain to the different elements or same element in different chemical environments.[20] XAS spectra can be recorded in both surface sensitive $(<5 \mathrm{~nm})$ using the total electron yield detection mode (TEY) or bulk sensitive $(<100 \mathrm{~nm})$ using the total fluorescence yield detection mode (FY).[21] The ability to project the electronic structure of the surface and bulk carries out important information on the chemical environments of the shell and interior of carbon nanocages.

We present herein a comprehensive X-ray spectroscopic study on the electronic structure of pristine $\mathrm{CNC}$ and the evolution of electronic structure upon nitrogen and phosphorus doping. Supported by extensive computational analysis (density functional theory (DFT) calculations), we demonstrate the doping effect on the electronic structure of carbon nanocages upon nitrogen and phosphorus doping. In addition, the chemical environment of the dopant atoms as well as dopant configuration are clearly revealed. Our findings emanate fingerprints on the local charge symmetry and chemical bonding surrounding the constituent atoms in the carbon nanomatrices. Further, such information carries great 
importance and sheds lights on the functional properties of the CNCs related to charge propagation and storage.

\section{Experimental}

\subsection{Synthesis of pristine and N-/P doped CNCs}

The pristine and N/P doped CNCs were synthesized by the MgO template method.[11,12] In brief, the pristine CNCs were prepared by the chemical vapor deposition method (CVD) using benzene as carbon source and annealing the $\mathrm{Mg}_{2}(\mathrm{OH})_{2} \mathrm{CO}_{3}$ at high temperature under the flow of $\mathrm{Ar}$ gas in the growth process. N-CNCs and P-CNCs were synthesized using similar method with pyridine and triphenylphosphine as the carbon source. Herein, all of the CNCs, N-CNCs and P-CNCs were synthesized under the same temperature $\left(700^{\circ} \mathrm{C}\right)$.

\subsection{Characterization}

A Kratos Axis Ultra ${ }^{D L D}$ XPS system equipped with a monochromatic Al Ka X-ray source was used for XPS experiments. Survey and high-resolution spectra were recorded at 80 and $20 \mathrm{eV}$ pass energy, respectively. The peak fitting of XPS was performed using XPS Peak 4.1 software. The XAS and XES spectra were recorded on BL6.3.1.2 and BL8.0.1 at the Advanced Light Source (ALS). The incident beam angle was $45^{\circ}$ with respect to the sample surface. The photon energy was calibrated using an HOPG standard reference sample with $\pi^{*}$ peak located at $285.5 \mathrm{eV}$.[18] The XES spectra were recorded using a Nordgren-type spectrometer,[22] and the elastic peak energy positions of the emission lines from the samples were used to calibrate the energy scale for the XES spectra. The energy resolution of XAS and XES were set to $0.1 \mathrm{eV}$ and $0.35 \mathrm{eV}$, respectively. The peak fitting of $\mathrm{N} K$-edge XAS was done using arctan function background method.

\subsection{Computational methods}

Theoretical analysis was conducted by simplifying the complex multilayered nanocages structure into 
single layered graphene structure. Such simplification is based on the fact that the X-ray absorption spectroscopy is a local probe technique which is not sensitive to the long range interaction between layers. Thus the interaction can be neglected and the simulation of nanocages can be simplified to graphene-based models. Such approximation has not only been proven by the comparison between graphite and graphene XAS experiments,[23] but also by theoretical simulations.[24] The ground-state calculations were done using DFT method implemented in the VASP package.[25] Large $11 \times 11$ supercells were employed. The Perdew-Burke-Ernzerhof (PBE) functional [26] in conjunction with the projector augmented wave (PAW) formalism [27] was used. The kinetic energy cutoff was set to $450 \mathrm{eV}$ with a $3 \times 3 \times 1$ Monkhorst-Pack [28] k-sampling scheme used for the first Brillouin zone. The Hellmann-Feynman force tolerance was set to $0.02 \mathrm{eV} / \AA$ for each atom. The excited X-ray absorption spectra were calculated by employing the ECH method.[29] Large hydrogen-terminated cluster models were constructed base on the ground-state calculation models. Detailed description of the method can be found elsewhere.[24],[30]

\section{Results and discussions}

\subsection{Chemical environments of carbon nanocages}

The structural properties (average cage size and shell thickness: $\sim 20 \mathrm{~nm}$, and $\sim 3 \mathrm{~nm}$, respectively), and the chemical composition of the pristine and doped $\mathrm{CNCs}(\mathrm{N} /[\mathrm{N}+\mathrm{C}]: \sim 10$ at.\%, and $\mathrm{P} /[\mathrm{P}+\mathrm{C}]: \sim 3$ at.\%) have been examined by the transmission electron microscopy (TEM) (Fig. 1) and X-ray photoelectron spectroscopy (XPS) (Fig. 2A). There are less than 10 layers in the cages, consistent with the thickness of the nanocage of about $3 \mathrm{~nm}$ measured by high-resolution TEM. There is no obvious difference in thickness between the N- and P-doped CNCs. Thickness and overall size (and pore size as well) will directly affect the surface area of cages,[17] which is related to the chemical activity. The relatively high concentration of $\mathrm{N}$ doping is probably due to the unique synthesis condition and the similar high concentration of $\mathrm{N}$ doping in other carbon systems are also reported, such as $\mathrm{N}$ doped CNT [31] and $\mathrm{N}$ 
doped HOPG[32].
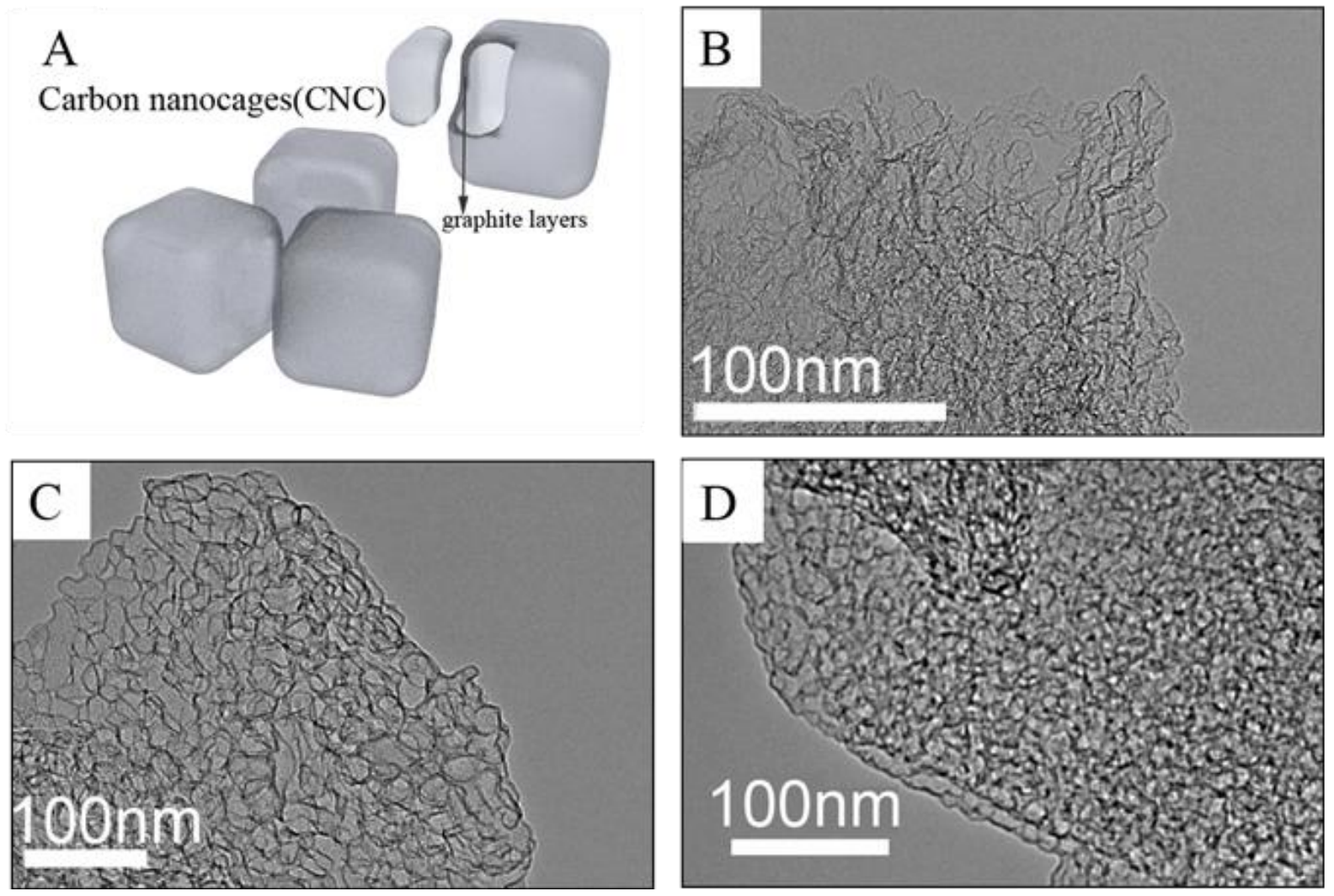

Fig. 1. (A)The schematic model of carbon nanocages. TEM images of pristine CNCs (B), N-CNCs (C) and P-CNCs (D).

Fig. 2B-2D present the $\mathrm{C} 1 s$ core-level binding energies of the pristine and N/P doped CNCs. The main peak at $c a .284 .6 \mathrm{eV}$ in Fig. $2 \mathrm{~B}$ reflecting $s p^{2} \mathrm{C}=\mathrm{C}$ bonding in the $\mathrm{CNCs}$ sample is found to shift towards the higher energies upon doping, $+0.35 \mathrm{eV}$ for N-CNCs (Fig. 2C) and $+0.15 \mathrm{eV}$ for P-CNCs (Fig. 2D) respectively, indicating a change in the local charge symmetry. Additional spectral features such as $\mathrm{C}-\mathrm{C} s p^{3}, \mathrm{C}-\mathrm{O}$ or C-N/C-P, C=O, COO, and $\mathrm{O}_{2} \mathrm{CO}$, shown as the insert of Fig. 2B-2D, have been identified following previous reported studies on the doped graphene.[33,34] 

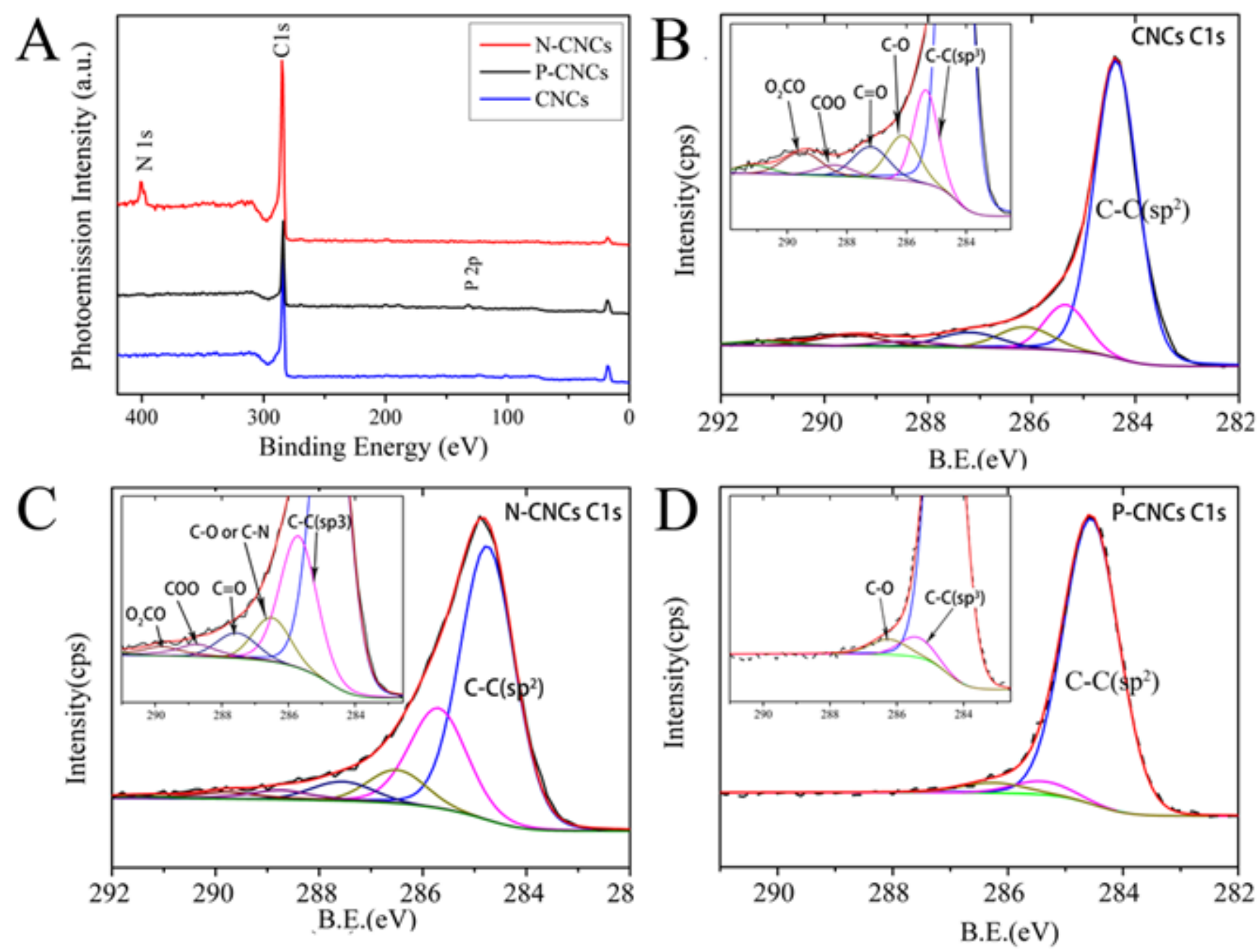

Fig. 2. The survey scan (A) and C $1 s$ high resolution XPS spectra of CNCs, N-CNCs and P-CNCs; (B) CNC C $1 s$ spectra; (C) N-CNCs C $1 s$ spectra; (D) P-CNCs C $1 s$ spectra. The deconvoluted results of C $1 s$ XPS were shown in the inset.

The $s p^{3}$ spectral feature in the pristine CNCs arises from the defected cage corner region of corresponding CNCs. The observed spectral broadening upon nitrogen doping can be attributed to an increase of the lattice disorder which typically arises from the change of chemical environments,[33] as previously reported in nitrogen doped CNTs/graphene at high impurity concentrations.[35-37] In addition to the core-hole screening in conductive materials, which unavoidably imparts line asymmetry in XPS spectra,[34] the increased asymmetry of the C $1 s$ peak of N-CNCs is attributed to a change in the 
conduction electrons due to the $\mathrm{C}-\mathrm{N}$ bond. In contrast to the N-CNCs, the P-CNCs show neither significant lattice disorder, nor existence of carbonate/carbonyl groups. The less lattice disorder in P-CNCs derives from the stabilization of the carbon matrix is sustained at low phosphorus concentrations. The absence of carbonate/carbonyl species in the C 1 s XPS of P-CNCs indicates that most of foreign oxygen atoms form $\mathrm{P}-\mathrm{O}$ bonding and $\mathrm{C}-\mathrm{O}$ bonding during the growth process. The higher structural ordering in P-CNCs than in N-CNCs is understandable. As from the XPS spectra, there are more bonding types in N-CNCs, in contrast to P-CNCs, which lead to more complex atomic structure with significant amount of defects or vacancies.

Nanomaterials with hollow structure typically have different chemical structures between shell and interior due to the synthesis condition.[38] To compare the chemical environments of the shell and interior of the nanocages, we recorded the C K-edge XAS spectra of pristine and doped CNCs in the TEY mode and TFY mode (shown in Fig. 3A). The spectral profile (TEY-XAS) in Fig. 3A resembles that of graphite,[18] which in line with the findings from the XPS analysis that carbon nanocage shell is mainly comprised of graphite-like layers structure. The peak(s) at $c a .285 .5 \mathrm{eV}$ and $292.0 \mathrm{eV}$ are attributed to the unoccupied $\mathrm{C}=\mathrm{C} \pi^{*}$ and $\mathrm{C}-\mathrm{C} \sigma^{*}$ states.[39] The features between the $\mathrm{C}=\mathrm{C} \pi^{*}$ and $\mathrm{C}-\mathrm{C} \sigma^{*}$ are assigned to the $\mathrm{C}-\mathrm{O} / \mathrm{C}-\mathrm{N}$ bonds. Observed reduction in the relative intensity of the $\pi^{*}$ peak and the spectral broadening in the N-CNCs arise from the substitution of carbon matrices by nitrogen atoms (Fig. 3B). In addition, the associated structural distortion, wherein the $\mathrm{N}$-CNCs manifest main $\pi^{*}$ peak of the lowest intensity, is plausibly due to higher impurity concentration (Fig. 3B). Interestingly, the spectral features of P-CNCs become well resolved upon phosphorus doping, which again demonstrates the stabilization of the carbon matrices upon phosphorus doping as phosphorus trends to form single bond with defected carbon.[40]

\subsection{Band structure}


The conduction band minimum $(\mathrm{CBM})$ has been extracted by extending the linear sections of the band (absorption edge) to where they intersect with the baseline. Upon doping, the $\pi^{*}$ shifts from $283.4 \mathrm{eV}$ to $283.6 \mathrm{eV}$ under nitrogen doping and from $283.4 \mathrm{eV}$ to $283.5 \mathrm{eV}$ under phosphorus doping, respectively (Fig. 3B). The shift in the edge of XAS is on account of the chemical bonding with N/P due to the doping effect.

In comparison with pristine and phosphorus doped $\mathrm{CNC}$, the most prominent difference in the N-CNCs is the peak labeled A' (at $\sim 286.4 \mathrm{eV}$ ) which is not seen in the samples with low-concentration nitrogen doped $\mathrm{CNT} /$ graphene.[41] It can be assigned to the $\mathrm{C}=\mathrm{N} \pi^{*}$ resonance in the pyridine-like ring of matrices, which also shows up in $\mathrm{C}_{3} \mathrm{~N}_{4}$ system.[42] It is consistent with the assignments of $\mathrm{C}=\mathrm{N}$ bond in XPS. The peak C ( 290.4 eV) appears in the FY-XAS while it disappears in the TEY-XAS. Such difference indicates that different chemical structures exist in the interior of nanocages comparing with the shell. The $\pi^{*}$ peak of carbonate is typically sharp, while we find no corresponding sharp feature in the resonant excited XES spectra at $290.4 \mathrm{eV}$ (shown in Fig. 3C). Therefore, it could correspond to the transitions from the symmetrical ground state to a core excitonic state, which is resulted from the electron-hole correlation as shown in diamond, graphite and graphene.[18,39,43] The exciton peak manifests that the carbon nanocages preserve the ordered interior atomic structure. This featured difference between TEY and TFY furnishes the evidences of electronic structure change for the interior of nanocages. The atomic structure of defected shell and ordered interior would improve the related properties, for example the electrode in supercapacitor and ORR catalyst.[11,12] 

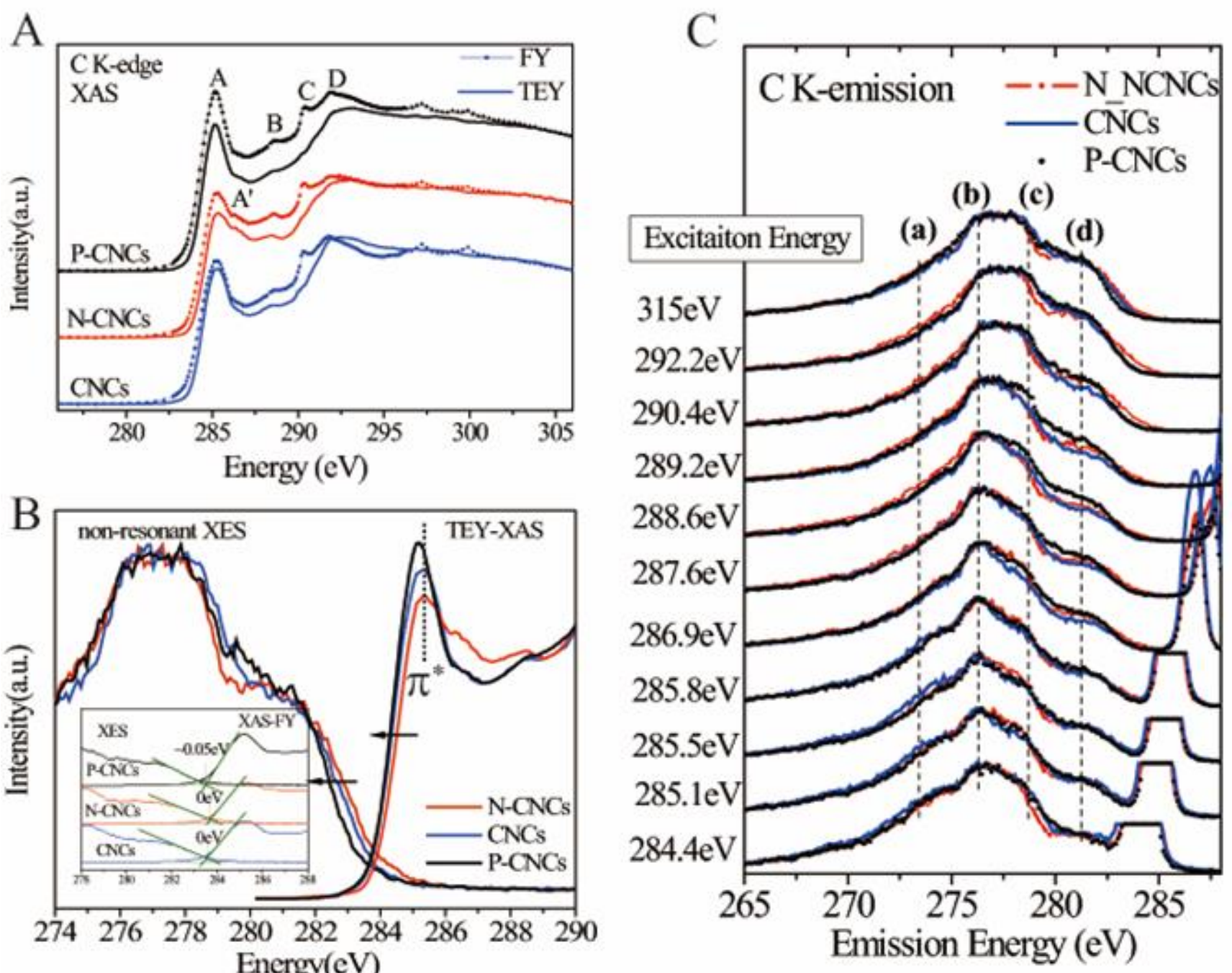

Fig. 3. C K-edge XAS and Ka XES spectra of CNCs, N-CNCs and P-CNCs: (A) C $K$-edge XAS spectra of CNCs, P-CNCs and N-CNCs recorded in TEY and TFY detection modes, and all spectra are normalized to both the pre-edge and post-edge. (B) The fine view of $\pi^{*}$ state in C $K$-edge XAS spectra (TEY mode) and C $K$-edge non-resonant XES (excited energy $h v=315 \mathrm{eV}$ ) of the CNCs, P-CNCs and N-CNCs. Band gap calculation from XAS-XES spectra is shown in the inset. (D). RXES spectra of CNCs, N-CNCs and P-CNCs. The elastic peak of spectra $(h v=285.5 \mathrm{eV})$ was cut off for plot.

The relative energy position of the valence band maximum (VBM) has been extracted via linear extrapolation of the valence band edge of non-resonant XES spectra (excitation energy at $315 \mathrm{eV}$ ) to the background baseline (see Fig. 3B). We observe a shift in the VBM towards lower energy upon the 
phosphorus doping (from $283.9 \mathrm{eV}$ to $283.45 \mathrm{eV}$ ) and towards higher energy upon nitrogen doping (from $283.9 \mathrm{eV}$ to $284.1 \mathrm{eV}$ ). With the VBM from XES, combined with the information about the relative energy position of the CBM from XAS in the carbon complexes, we could project the variation of band gap $\left(\mathrm{E}_{\mathrm{g}}\right)$ of CNCs. The zero Eg value manifests the metal-like properties of CNCs and N-CNCs. The non-zero band gap $(0.05 \mathrm{eV})$ means that phosphorus opens the band gap of carbon nanocages.

The spectral change of the X-ray emission spectra as a function of excitation energy from $285.5 \rightarrow 315$ $\mathrm{eV}$ (Fig. 3C) relates to the band structure of carbon nanocages. Similar line-shape in the non-resonant XES $(h v=315 \mathrm{eV})$ of all three types of nanocages indicates the analogous DOSs in the valence state. The pre-edge shoulder feature (d) at $c a .282 \mathrm{eV}$ and the main peak (b) at $c a .276 \mathrm{eV}$ arise from $\pi$ and $\sigma$ states of carbon matrices, respectively.[43,44] At excitation energies $\geq 286.9 \mathrm{eV}(\mathrm{C}=\mathrm{N})$, the edge of feature (d) shifts towards higher energy upon nitrogen doping, which is attributed to the contribution from $\mathrm{C}=\mathrm{N} P_{z}$ states. The upshift of feature (d) can be explained by the deeper core level of carbon atoms in N-CNCs, because carbon is bonded to nitrogen with the lower core level energies that is evident from $\mathrm{C} 1 \mathrm{~s}$ XPS.[42] The relative intensity of peak (a) is more pronounced in CNCs at excitation energy $285.5 \mathrm{eV}$ $(C=C)$. Also, when excitation energies are higher than $285.5 \mathrm{eV}\left(\mathrm{C}=\mathrm{C} \pi^{*}\right)(286.9 \mathrm{eV}-290.4 \mathrm{eV})$, peak (d) in the CNCs show the lowest intensity. The lower peak (a) and (d) suggest that the content of $\mathrm{C}=\mathrm{C} s p^{2}$ orbitals is lower than the doped cages. It is resulted from the $s p^{3}$ orbitals in the defected shell region of CNCs.

The XES spectra excited at energies of $290.4 \mathrm{eV}$ and $292.2 \mathrm{eV}$ show great resemblance, which indicates that the post-edge feature (c) of C $K$-edge XAS (Fig. 3A) is not attributed to the carbonate species.[45] In addition, feature (c) becomes more prominent and intensified at higher excitation energies ( $\geq 290.4 \mathrm{eV}$ ). The increasing feature (a) in the N-CNCs can be partially attributed to the $\sigma$ state of carbon bonded with foreign atoms (N/O/P), and the feature (c) can be attributed to the $\pi$ states of these carbon 
atoms bonding with foreign atoms.[44,46]

\subsection{N/P doping configuration}

In a doped carbon system, the dopant normally activates its surrounding carbon atoms leading to improved performance in practical application such as in ORR reactivity.[6,7,10,47] As shown in Fig. 4,

$\mathrm{N} 1 s$ and $\mathrm{P} 2 p$ XPS give the description of N/P chemical environments in nanocages. The $\mathrm{N} 1 s$ high-resolution XPS can be de-convoluted to pyridine-like $\mathrm{N}$ at $398.4 \mathrm{eV}$, pyrrole-like $\mathrm{N}$ at $399.6 \mathrm{eV}$, and graphite-like $\mathrm{N}$ at $401.1 \mathrm{eV}$.[41] Phosphorus doping sites are also investigated by high-resolution $\mathrm{P} 2 p$ XPS, displayed in Fig 4B. It reveals large proportion of P-C bonding (132.7 eV) and P-O bonding (133.8 eV) in P-CNCs.[47] Unlike the nitrogen doping in which nitrogen atoms can substitute the carbon atoms in the matrices and form various configurations, phosphorus atom is inclined to stand out of the planar matrices and form bonds with carbon atoms.[40] Therefore, combining with the results from C $1 s$ XPS and $\mathrm{C} K$-edge XAS, the low content of phosphorus atoms will exist in graphite-like configuration in carbon matrices of P-CNCs, which could support the higher degree of ordering in the P-CNCs. Meanwhile, the formation of $\mathrm{P}-\mathrm{O}$ bonds is helpful to avoid disordering the carbon matrices.
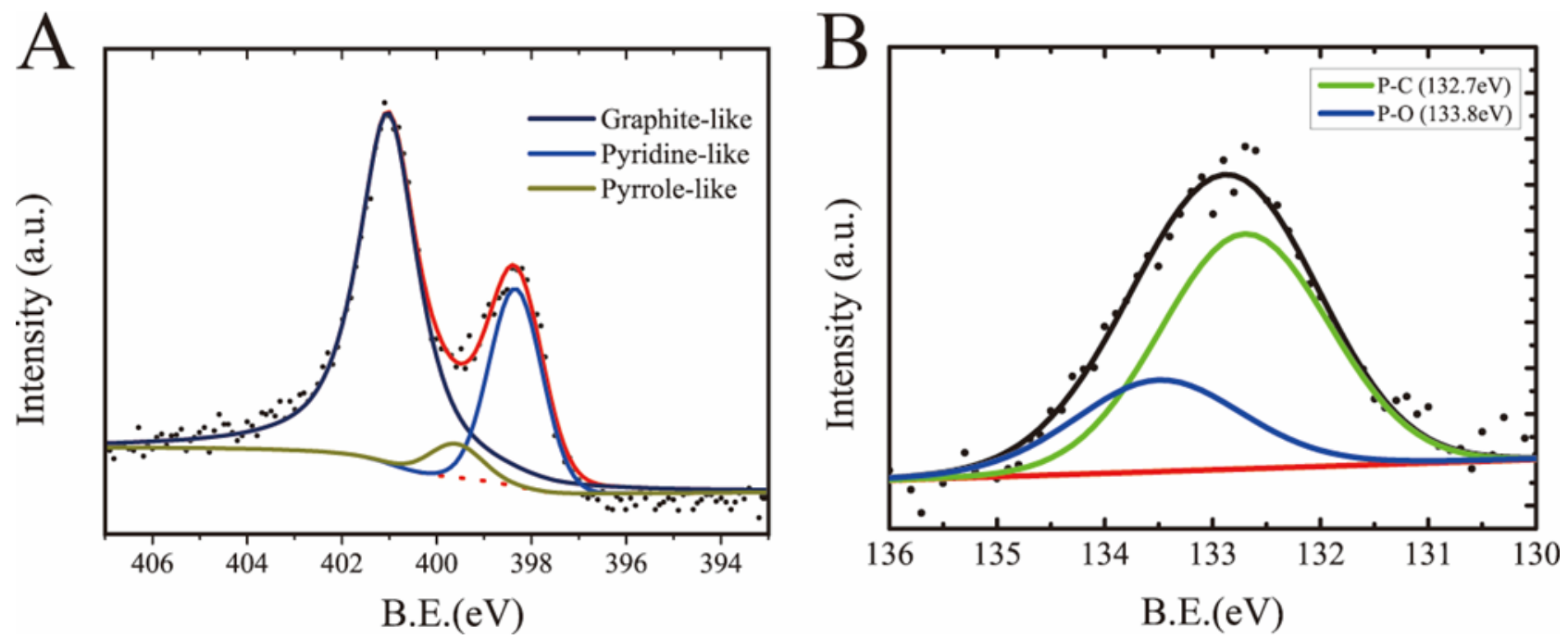

Fig. 4. $\mathrm{N} 1 s$ XPS of N-CNCs (A) and P $2 p$ XPS of P-CNCs (B) with fitting peaks. 
Based on N 1s XPS results, the three plausible doping configurations of N-CNCs: pyridine-like N, pyrrole-like $\mathrm{N}$ and graphite-like $\mathrm{N}$, are presented in Fig. 5A.[41] Pyridine-like $\mathrm{N}$ atom has a 6-member ring structure bonding with two neighboring carbon atoms, while pyrrole-like $\mathrm{N}$ atom exists in a 5-member ring structure bonding with two neighboring carbon atoms. For the graphite-like $\mathrm{N}$ atom, a nitrogen atom substitutes a carbon atom in the graphene layer. Compared to $\mathrm{N} 1 s$ XPS, N K-edge XAS has better resolution in distinguishing different nitrogen doping sites. From the N $K$-edge XAS in Fig. 5B,

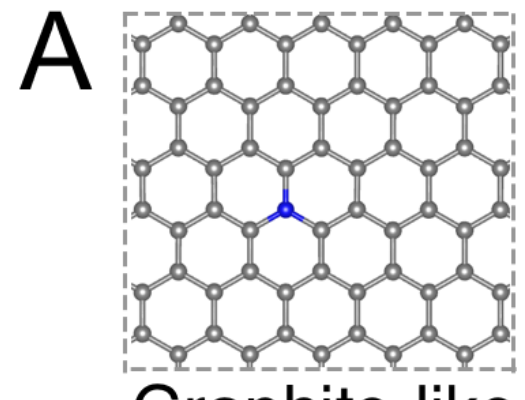

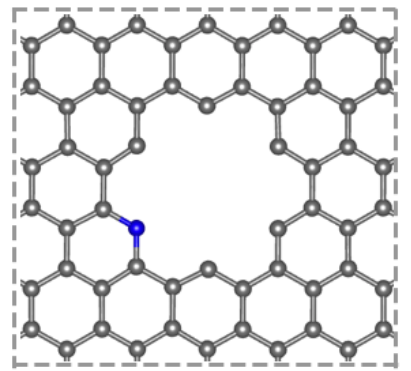

Pyridine-like

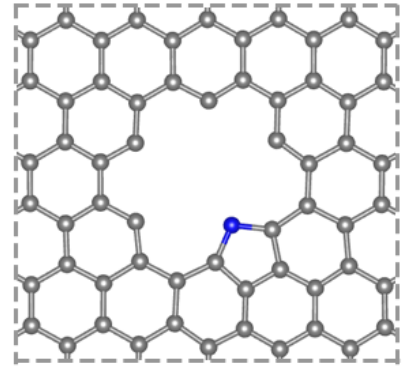

Pyrrole-like
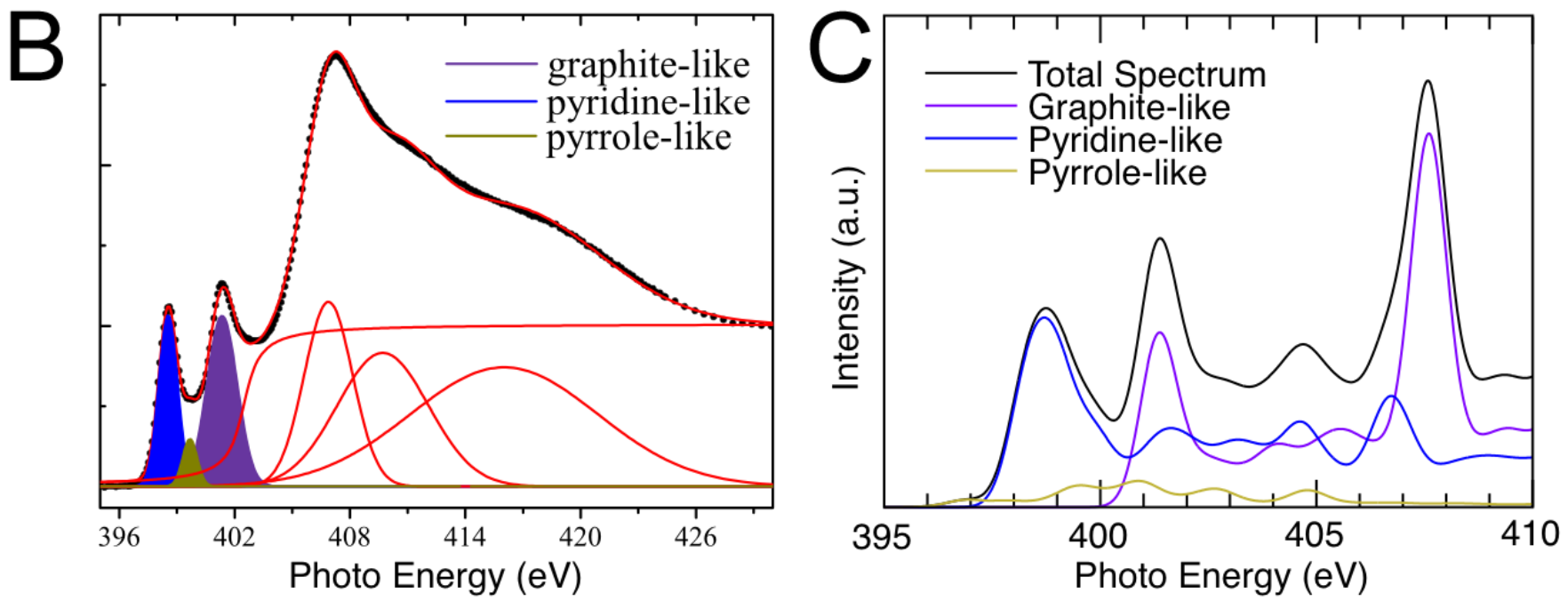

Fig. 5. (A) Three nitrogen doping configurations of N-CNCs: graphite-like, pyridine-like, and pyrrole-like; (B) N $1 s$ XAS of N-CNCs with the fitting peaks using acting background method; (C) Calculated N 1s XAS spectra with related nitrogen species: graphite-like, pyridine-like, and pyrrole-like N. The contribution from graphite-like, pyridine-like and pyrrole-like doping configurations are plotted in purple, blue and brown respectively. 
we can see two obvious main peaks at $\sim 398.5 \mathrm{eV}$ and $\sim 401.3 \mathrm{eV}$, which can be assigned to $\pi^{*}$ state of pyridine-like and graphite-like $\mathrm{N}$, respectively.[35,48,49] The broaden peak above $405 \mathrm{eV}$ is associated with the $\sigma^{*}$ resonance of $\mathrm{C}-\mathrm{N}$ environment. Herein, the nitrogen doping in the graphitic carbon structure show two main different structures, which is also in agreement with previous XAS studies of $\mathrm{CN}_{\mathrm{x}}$ films,[50] $\mathrm{N}$ doped CNTs [51] and $\mathrm{N}$ doped graphene.[52] Meanwhile, the small percentage of pyrrole-like $\mathrm{N}$ at $399.7 \mathrm{eV}$ could be resolved by the peak fitting of XAS using act-angle function background method. The positions of the three peaks match well with the $\mathrm{N} 1 s$ high-resolution XPS results.

\subsection{DFT calculation on doping effect}

Theoretically, the $\mathrm{N}$ doped CNCs model was simplified as $\mathrm{N}$ doped graphene to simulate the $\mathrm{N}$ K-edge XAS spectra shown in Fig. 5C, considering the interaction between layers is much less than that from the intra-layer bonding. As different doping configurations show different spectral features in both energy and intensity, to compare with experiment spectra properly, we generate the final spectral shape by making a sum of the spectra from the configurations of graphite-like, pyridine-like and pyrrole-like with a concentration ratio of 1:0.7:0.1. Unlike pyrrole-like doping which presents a very broaden $\pi^{*}$ feature, the graphite-like and pyridine-like doping configurations show very clear $\pi^{*}$ peaks. The $\pi^{*}$ peak of pyridine-like locates at $398.6 \mathrm{eV}$, which is $2.7 \mathrm{eV}$ lower than that of graphite-like, agreeing well with the experimental value $(\sim 2.8 \mathrm{eV})$. The calculated spectra confirm the successful synthesis of the dominant graphite-like and pyridine-like doping configuration. The broadened $\pi^{*}$ feature of pyrrole-like doping indicates that this kind of doping concentration is very low in current sample synthesis process, otherwise the $\pi^{*}$ feature of the total spectrum will be much broader rather than two distinguishable peaks. The results match with the low area ratio in the fitting results of XAS and XPS. The $\sigma^{*}$ peak locating at 407.6 $\mathrm{eV}$ is from the graphite-like doping configuration, which is believed to be the main contributor of the $\sigma^{*}$ 
peak after $405 \mathrm{eV}$. It is worthy to mention that the intensity of each peak depends on the concentration of each doping configuration, so a different doping level will lead to different peak intensity. However, the contribution from each doping configuration can always be distinguishable. Combining the simulation and experiment, the nitrogen chemical environment can be obtained, which supports the understanding of the properties of N-CNCs.

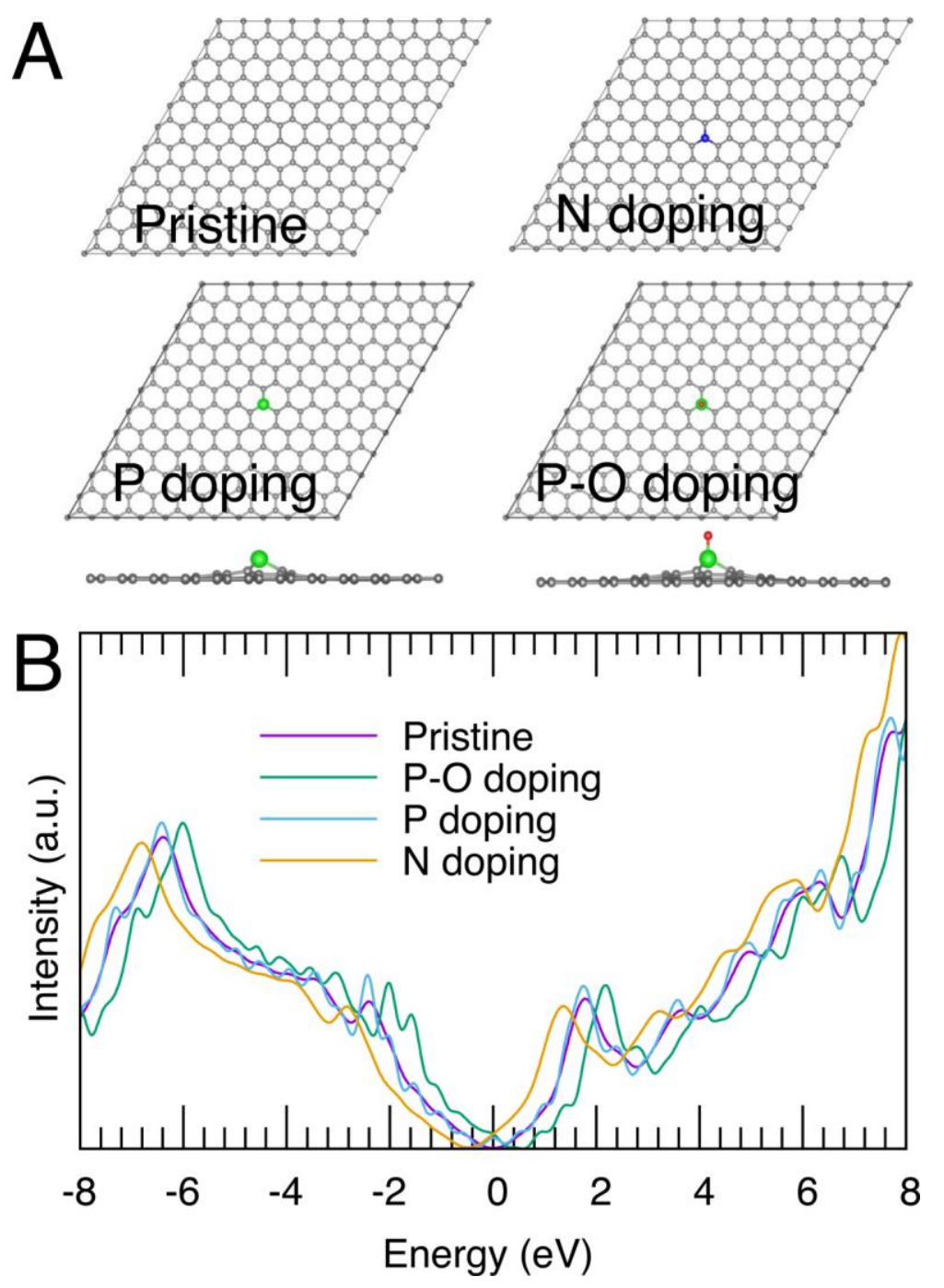

Fig. 6. (A) Model structures of pristine and $\mathrm{N}$ and $\mathrm{P}$ (with and without $\mathrm{O}$ ) doped graphene layer with the side view of $\mathrm{P}$ doping shown in the bottom panel, and corresponding density of state (B). Color scheme for atoms: grey, C; blue, N; red, O; and green, P.

In experiments, N-CNCs show better performance in ORR reactions comparing to CNCS.[11] The 
reason is probably lying in their electronic structure near the Fermi level for P-CNCs. Here we calculated the density of states (DOS) for all of P/N-CNCs and pristine CNC as shown in Fig. 6. For P-CNCs, both single P and P-O types of configuration are considered. The graphite-like configuration were chosen as the doping model structures shown in Fig. 6A, as that is the majority components in both $\mathrm{P}$ and $\mathrm{N}$ doped CNCs. The N doping does not change the planar geometry, while the P doping shows differently that the $\mathrm{P}$ atom stands out of the carbon plane bonding with the neighboring carbon atoms. From the DOS results shown in Fig. 6B, it is clear that the $\mathrm{N}$ serves as n-type doping, while the P-O serves as p-type doping. The density of states of $\mathrm{P}$ doping without oxygen show similar profile as for pristine CNCs, except a small defect state appearing at the Fermi level. The behaviors of P and P-O doped CNCs are similar to those doped carbon nanotubes.[53] Considering the opposite behavior of $\mathrm{N}$ doping and P-O doping to the density of states, and the higher catalysis performance of $\mathrm{N}$-CNCs than pristine CNCs in the ORR reaction studied previously,[11] it can be expected that the P-CNCs (P doping and P-O doping) may not be a good electrochemical catalyst candidate as N-CNCs.

\section{Conclusion}

The pristine and N/P doped CNCs have been extensively examined by soft X-ray spectroscopies, including $\mathrm{C} / \mathrm{N}$ 1s and $\mathrm{P} 2 \mathrm{p}$ XPS, $\mathrm{N}$ and $\mathrm{C}$ K-edge XAS, and $\mathrm{C}$ K-edge XES/RXES. The chemical environments of doping sites are clearly elucidated. The N-doping results in three configurations of graphite-like, pyridine-like and pyrrole-like wherein the latter one is lower in concentration than the other two. The phosphorus doping forms mostly P-C $s p^{3}$ covalent bond with the neighboring carbon, leading to more ordered atomic structure than the nitrogen doping. Furthermore, the interior of nanocages appears highly ordered geometry structure. The XAS spectra in TEY and TFY modes reveal that the shell and interior of nanocages show difference in electronic structure, and the difference is independent from the doping types. Both experimental spectroscopy and DOS calculation show greater modification of 
electronic structure for nitrogen doping than for phosphorus doping, which may be an indication for the higher performance in ORR reactions for the nitrogen doping. A comprehensive investigation of the electronic structures for pristine/doped nanocages provides fundamental knowledge of the N/P-CNCs and CNCs, which is important for the future improvements on the related applications.

\section{Acknowledgments}

The work is supported by Natural Science Foundation of China (NSFC) (Grant No. 91333112, U1432249), the Priority Academic Program Development of Jiangsu Higher Education Institutions. This is also a project supported by Collaborative Innovation Center of Suzhou Nano Science \& Technology and sponsored by Qing Lan Project. The Advanced Light Source is supported by the Director, Office of Science, Office of Basic Energy Sciences, of the U.S. Department of Energy under Contract No. DE-AC02-05CH11231. The Swedish National Infrastructure for Computing (SNIC) and the National Energy Research Scientific Computing Center (NERSC) are acknowledged for the computational resources.

\section{Reference:}

[1] Collins PG, Bradley K, Ishigami M, Zettl A. Extreme oxygen sensitivity of electronic properties of carbon nanotubes. Science 2000;287(5459):1801-4.

[2] Zhu Y, Murali S, Cai W, Li X, Suk JW, Potts JR, et al. Graphene and graphene oxide: Synthesis, properties, and applications. Adv Mater 2010;22(35):3906-24.

[3] Ji XL, Lee KT, Nazar LF. A highly ordered nanostructured carbon-sulphur cathode for lithium-sulphur batteries. Nat Mater 2009;8(6):500-6.

[4] Yoo E, Kim J, Hosono E, Zhou H-S, Kudo T, Honma I. Large reversible Li storage of graphene 
nanosheet families for use in rechargeable lithium ion batteries. Nano Lett 2008;8(8):2277-82.

[5] Landi BJ, Ganter MJ, Cress CD, DiLeo RA, Raffaelle RP. Carbon nanotubes for lithium ion batteries. Energy Environ Sci 2009;2(6):638-54.

[6] Gong K, Du F, Xia Z, Durstock M, Dai L. Nitrogen-doped carbon nanotube arrays with high electrocatalytic activity for oxygen reduction. Science 2009;323(5915):760-4.

[7] Zhao Y, Yang L, Chen S, Wang X, Ma Y, Wu Q, et al. Can boron and nitrogen co-doping improve oxygen reduction reaction activity of carbon nanotubes? J Am Chem Soc 2013;135(4):1201-4.

[8] Liang Y, Li Y, Wang $\mathrm{H}$, Zhou J, Wang J, Regier T, et al. $\mathrm{Co}_{3} \mathrm{O}_{4}$ nanocrystals on graphene as a synergistic catalyst for oxygen reduction reaction. Nat Mater 2011;10(10):780-6.

[9] Jiang S, Ma Y, Jian G, Tao H, Wang X, Fan Y, et al. Facile construction of Pt-Co/CN $\mathrm{C}_{\mathrm{x}}$ nanotube electrocatalysts and their application to the oxygen reduction reaction. Adv Mater 2009;21(48):4953.

[10] Yang L, Jiang S, Zhao Y, Zhu L, Chen S, Wang X, et al. Boron-doped carbon nanotubes as metal-free electrocatalysts for the oxygen reduction reaction. Angew Chem, Int Ed 2011;50(31):7132-5.

[11] Chen S, Bi J, Zhao Y, Yang L, Zhang C, Ma Y, et al. Nitrogen-doped carbon nanocages as efficient metal-free electrocatalysts for oxygen reduction reaction. Adv Mater 2012;24(41):5593-7.

[12]Xie K, Qin X, Wang X, Wang Y, Tao H, Wu Q, et al. Carbon nanocages as supercapacitor electrode materials. Adv Mater 2012;24(3):347-52.

[13]Lyu Z, Xu D, Yang L, Che R, Feng R, Zhao J, et al. Hierarchical carbon nanocages confining high-loading sulfur for high-rate lithium-sulfur batteries. Nano Energy 2015;12:657-65.

[14]Lyu Z, Yang L, Xu D, Zhao J, Lai H, Jiang Y, et al. Hierarchical carbon nanocages as high-rate anodes for Li-and Na-ion batteries. Nano Research 2015;8(11):3535-43.

[15]Zhou M, Wang H-L, Guo S. Towards high-efficiency nanoelectrocatalysts for oxygen reduction through engineering advanced carbon nanomaterials. Chem Soc Rev 2016;DOI: 10.1039/C5CS00414D. 
[16] Yan J, Wang Q, Wei T, Fan Z. Recent advances in design and fabrication of electrochemical supercapacitors with high energy densities. Advanced Energy Materials 2014;4(4):1300816.

[17]Zhao J, Lai H, Lyu Z, Jiang Y, Xie K, Wang X, et al. Hydrophilic hierarchical nitrogen-doped carbon nanocages for ultrahigh supercapacitive performance. Adv Mater 2015;27(23):3541-5.

[18] Ma Y, Skytt P, Wassdahl N, Glans P, Guo J, Nordgren J. Core excitons and vibronic coupling in diamond and graphite. Phys Rev Lett 1993;71(22):3725-8.

[19]Zhong J, Zhang H, Sun X, Lee ST. Synchrotron soft X - ray absorption spectroscopy study of carbon and silicon nanostructures for energy applications. Adv Mater 2014;26(46):7786-806.

[20]Guo J. Synchrotron radiation, soft-X-ray spectroscopy and nanomaterials. Int J Nanotechnol 2004;1(1-2):193-225.

[21]Liu L, Sham T-K, Han W, Zhi C, Bando Y. X-ray excited optical luminescence from hexagonal boron nitride nanotubes: Electronic structures and the role of oxygen impurities. ACS Nano 2011;5(1):631-9.

[22]Nordgren J, Bray G, Cramm S, Nyholm R, Rubensson J-E, Wassdahl N. Soft X-ray emission spectroscopy using monochromatized synchrotron radiation. Rev Sci Instrum 1989;60(7):1690-6.

[23]Papagno M, Rodríguez AF, Girit Ç, Meyer J, Zettl A, Pacilé D. Polarization-dependent C (K) near-edge X-ray absorption fine-structure of graphene. Chem Phys Lett 2009;475(4):269-71.

[24]Hua W, Gao B, Li S, Ågren H, Luo Y. X-ray absorption spectra of graphene from first-principles simulations. Phys Rev B 2010;82(15):155433.

[25]Kresse G, Furthmüller J. Efficient iterative schemes for ab initio total-energy calculations using a plane-wave basis set. Phys Rev B 1996;54(16):11169.

[26]Perdew JP, Burke K, Ernzerhof M. Generalized gradient approximation made simple. Phys Rev Lett 1996;77(18):3865.

[27]Kresse G, Joubert D. From ultrasoft pseudopotentials to the projector augmented-wave method. Phys 
Rev B 1999;59(3):1758.

[28]Monkhorst HJ, Pack JD. Special points for brillouin-zone integrations. Phys Rev B 1976;13(12):5188.

[29]Plashkevych O, Privalov T, Ågren H, Carravetta V, Ruud K. On the validity of the equivalent cores approximation for computing X-ray photoemission and photoabsorption spectral bands. Chem Phys 2000;260(1):11-28.

[30]Li X, Hua W, Guo J, Luo Y. Electronic structure of nitrogen-doped graphene in the ground and core-excited states from first-principles simulations. J Phys Chem C 2015;119(29):16660-6.

[31]Li X, Liu J, Zhang Y, Li Y, Liu H, Meng X, et al. High concentration nitrogen doped carbon nanotube anodes with superior $\mathrm{Li}^{+}$storage performance for lithium rechargeable battery application. $\mathrm{J}$ Power Sources 2012;197:238-45.

[32]Guo D, Shibuya R, Akiba C, Saji S, Kondo T, Nakamura J. Active sites of nitrogen-doped carbon materials for oxygen reduction reaction clarified using model catalysts. Science 2016;351(6271):361-5. [33]Reddy ALM, Srivastava A, Gowda SR, Gullapalli H, Dubey M, Ajayan PM. Synthesis of nitrogen-doped graphene films for lithium battery application. ACS Nano 2010;4(11):6337-42.

[34]Estrade-Szwarckopf H. XPS photoemission in carbonaceous materials: A "defect" peak beside the graphitic asymmetric peak. Carbon 2004;42(8-9):1713-21.

[35] Maldonado S, Morin S, Stevenson KJ. Structure, composition, and chemical reactivity of carbon nanotubes by selective nitrogen doping. Carbon 2006;44(8):1429-37.

[36]Ronning C, Feldermann H, Merk R, Hofsäss H, Reinke P, Thiele JU. Carbon nitride deposited using energetic species: A review on XPS studies. Phys Rev B 1998;58(4):2207.

[37] Wei D, Liu Y, Wang Y, Zhang H, Huang L, Yu G. Synthesis of N-doped graphene by chemical vapor deposition and its electrical properties. Nano Lett 2009;9(5):1752-8. 
[38]Zhou J, Wang J, Liu H, Banis MN, Sun X, Sham T-K. Imaging nitrogen in individual carbon nanotubes. The Journal of Physical Chemistry Letters 2010;1(11):1709-13.

[39]Zhang L, Pollak E, Wang W-C, Jiang P, Glans P-A, Zhang Y, et al. Electronic structure study of ordering and interfacial interaction in graphene/Cu composites. Carbon 2012;50(14):5316-22.

[40]Denis PA. When noncovalent interactions are stronger than covalent bonds: Bilayer graphene doped with second row atoms, aluminum, silicon, phosphorus and sulfur. Chem Phys Lett 2011;508(13):95-101.

[41]Zhong J, Deng J-J, Mao B-H, Xie T, Sun X-H, Mou Z-G, et al. Probing solid state N-doping in graphene by X-ray absorption near-edge structure spectroscopy. Carbon 2012;50(1):335-8.

[42]Hellgren N, Guo JH, Luo Y, Sathe C, Agui A, Kashtanov S, et al. Electronic structure of carbon nitride thin films studied by X-ray spectroscopy techniques. Thin Solid Films 2005;471(1-2):19-34.

[43]Skytt P, Glans P, Mancini DC, Guo JH, Wassdahl N, Nordgren J, et al. Angle-resolved soft-X-ray fluorescence and absorption study of graphite. Phys Rev B 1994;50(15):10457-61.

[44]Endo K, Koizumi S, Otsuka T, Suhara M, Morohasi T, Kurmaev EZ, et al. Analysis of XPS and XES of diamond and graphite by DFTcalculations using model molecules. J Comput Chem 2001;22(1):102-8. [45]Augustsson A, Herstedt M, Guo J-H, Edström K, Zhuang G, Ross Jr P, et al. Solid electrolyte interphase on graphite Li-ion battery anodes studied by soft X-ray spectroscopy. Phys Chem Chem Phys 2004;6(16):4185-9.

[46]Hunt A, Dikin DA, Kurmaev EZ, Boyko TD, Bazylewski P, Chang GS, et al. Epoxide speciation and functional group distribution in graphene oxide paper-like materials. Adv Funct Mater 2012;22(18):3950-7.

[47] Yang D-S, Bhattacharjya D, Inamdar S, Park J, Yu J-S. Phosphorus-doped ordered mesoporous carbons with different lengths as efficient metal-free electrocatalysts for oxygen reduction reaction in 
alkaline media. J Am Chem Soc 2012;134(39):16127-30.

[48]Choi HC, Park J, Kim B. Distribution and structure of $\mathrm{N}$ atoms in multiwalled carbon nanotubes using variable-energy X-ray photoelectron spectroscopy. J Phys Chem B 2005;109(10):4333-40.

[49]Wang Y, Shao Y, Matson DW, Li J, Lin Y. Nitrogen-doped graphene and its application in electrochemical biosensing. ACS Nano 2010;4(4):1790-8.

[50]Hellgren N, Guo J, Sathe C, Agui A, Nordgren J, Luo Y, et al. Nitrogen bonding structure in carbon nitride thin films studied by soft X-ray spectroscopy. Appl Phys Lett 2001;79(26):4348-50.

[51]Kundu S, Nagaiah TC, Xia W, Wang Y, Dommele SV, Bitter JH, et al. Electrocatalytic activity and stability of nitrogen-containing carbon nanotubes in the oxygen reduction reaction. J Phys Chem C 2009;113(32):14302-10.

[52]Usachov D, Vilkov O, Gruneis A, Haberer D, Fedorov A, Adamchuk VK, et al. Nitrogen-doped graphene: Efficient growth, structure, and electronic properties. Nano Lett 2011;11(12):5401-7.

[53] Araujo JR, Silva AM, Gouvêa CP, Lopes ES, Santos RA, Terrazos L, et al. Phosphorous bonding in single wall carbon nanotubes studied by X-ray photoelectron spectroscopy and DFT calculations. Carbon 2016;99:1-7. 